\title{
Avaliação radiográfica da altura mandibular anterior após vestibuloplastia por inversão de retalhos: estudo em humanos ${ }^{\dagger}$ Radiographic evaluation of the anterior height of the mandible after vestibuloplasty through the lipswitch technique: study in humans
}

\author{
Cássio Edvard SVERZUT* \\ Mário Francisco Real GABRIELLI** \\ Eduardo Hochuli VIEIRA** \\ Alexander Tadeu SVERZUT***
}

\begin{abstract}
SVERZUT, C. E.; GABRIELLI, M. F. R.; VIEIRA, E. H.; SVERZUT, A. T. Avaliação radiográfica da altura mandibular anterior após vestibuloplastia por inversão de retalhos: estudo em humanos. Pesqui Odontol Bras, v. 15, n. 2, p. 133-137, abr./jun. 2001.
\end{abstract}

\begin{abstract}
A proposta do presente estudo foi de analisar, através de análise cefalométrica por telerradiografias de perfil, a altura óssea na região mandibular anterior após a realização de vestibuloplastia pela técnica de inversão de retalhos. Foram avaliados 13 pacientes (8 mulheres e 5 homens), com média igual a 54,3 anos que apresentavam, em média, 25,01 mm de altura mandibular anterior. As medidas foram obtidas utilizando-se traçado cefalométrico e comparando-se o período pré-operatório com os períodos pós-operatórios, que consistiram de 30, 90 e 180 dias. Durante o período analisado, houve uma redução da altura mandibular anterior, estatisticamente não significante, de 0,6 mm.
\end{abstract}

UNITERMOS: Reabsorção óssea; Perda óssea alveolar; Vestibuloplastia.

\section{INTRODUÇÃO}

O tecido ósseo, em todos os indivíduos, aumenta durante a fase de crescimento, ocorrendo um pico aos 20 anos de idade, estando este pico na dependência de fatores genéticos primariamente ${ }^{5,23}$, com potencial contribuição do cálcio, dieta e exercícios durante a infância e puberdade ${ }^{19}$. Porém, começa a ocorrer um declínio após os 30 anos de idade, exibindo uma acelerada perda em mulheres, 5 a 10 anos após a menopausa ${ }^{19,23}$. Após a formação óssea, ou seja, o término da fase de crescimento, ocorre um processo de remodelação óssea, contínuo por toda a vida, sob uma variedade de influências como, por exemplo, idade do organismo, localização, estresse e atividade metabólica ${ }^{3,5}$. Nas condições fisiológicas normais, essa remodelação é regulada primariamente pela estimulação $0^{3,5,23,28}$.

Especificamente na cavidade bucal, a falta de estímulo pode ocorrer com a perda do elemento dentário, fenômeno que irá se traduzir em perda óssea gradativa do processo alveolar ${ }^{17}$. $\mathrm{HOWE}^{11}$ (1965) encontrou diminuição no trabeculado e perda óssea em largura e altura no processo alveolar após exodontias. Esse fenômeno é chamado por alguns autores de atrofia óssea por desuso ${ }^{10,17}$.

A perda óssea alveolar é quase generalizada, mas com grandes variações individuais ${ }^{3,16}$. A taxa de redução do rebordo mandibular é inicialmente alta, de aproximadamente $12 \mathrm{~mm} /$ ano durante a fase imediata pós-extração e nivela-se estimadamente a $0,2 \mathrm{~mm} /$ ano após $2 \operatorname{anos}^{10}$. A redução média em altura do rebordo anterior é de 0,12 a $0,40 \mathrm{~mm} / \mathrm{ano}^{2,24,26}$. Esse processo é crônico, progressivo, irreversivel e acumulativo ${ }^{2,10}$.

Outros fatores podem estar presentes e influenciar o processo de reabsorção, como carga adversa da prótese muco-suportada, alterações secundárias à inflamação dos tecidos moles da área cha-

\footnotetext{
†Parte da Tese de Mestrado do Curso de Pós-Graduação em Odontologia pela Faculdade de Odontologia de Araçatuba da UNESP.

*Professor da Disciplina de Cirurgia; ${ }^{* *}$ Graduando em Odontologia - Faculdade de Odontologia de Ribeirão Preto da USP.

** Professores da Disciplina de Cirurgia da Faculdade de Odontologia de Araraquara da UNESP.
} 
SVERZUT, C. E.; GABRIELLI, M. F. R.; VIEIRA, E. H.; SVERZUT, A. T. Avaliação radiográfica da altura mandibular anterior após vestibuloplastia por inversão de retalhos: estudo em humanos. Pesqui Odontol Bras, v. 15, n. 2, p. 133-137, abr./jun. 2001.

peável ${ }^{10,21}$, função mandibular alterada, alterações vasculares, procedimentos cirúrgicos que requerem elevação muco-periostal ${ }^{1}$ e o uso de prótese muco-suportada ${ }^{10,19}$.

A perda óssea alveolar difere na maxila e na mandíbula. Essa diferença é manifestada de forma quantitativa, qualitativa e espacial ${ }^{1}$. O osso alveolar mandibular sofre uma perda quatro vezes maior do que o maxilar, $3,10,13,24$. Outro fator importante a considerar é que a maxila possui uma área chapeável 1,8 vezes maior que a mandibula ${ }^{3}$. Portanto, forças compressivas, que podem incrementar o processo de reabsorção, são maiores na mandíbula ${ }^{3,24}$.

Os protesistas muito freqüentemente defrontam-se com um rebordo residual mandibular extensamente reabsorvido, que pode ter um vestíbulo bucal raso e inserção alta do músculo mentoniano, em relação à crista do rebordo, podendo, com a sua contração, causar o desaparecimento do sulco vestibular ${ }^{7}$. De fato, grande parcela dos pacientes com atrofia mandibular tem uma área mínima de apoio para a prótese e a cirurgia pré-protética é um método valioso para aumentá-la ${ }^{15}$.

No sulco vestibular inferior, os procedimentos pré-protéticos são planejados para deslocar as origens do músculo mentoniano e músculos adjacentes do lábio e do mento ${ }^{11}$. Atualmente, entre os procedimentos utilizados em pacientes desdentados, está a técnica ilustrada por PICHLER; TRAUNER ${ }^{22}$ (1930) e descrita por HOWE ${ }^{11}$ (1965). Esta técnica é freqüentemente chamada de Kazanjian modificada, pois utiliza principios estabelecidos por este autor ${ }^{14}$ em 1924, inversão de retalhos ${ }^{14}$ ou transposição de retalhos ${ }^{18}$. Porém, é importante que o cirurgião conheça os diversos métodos de vestibuloplastia atualmente aceitos, uma vez que não há um método único que seja universalmente aplicá$\mathrm{vel}^{14}$. Convém salientar que, nos dias atuais, esta técnica vem sendo associada, concomitante ou não, com a colocação de implantes osseointegra$\operatorname{dos}^{15,20,25}$.

A atrofia mandibular é uma das principais indicações para vestibuloplastia, conjugada ou não com implantes osseointegrados, e tem havido interesse crescente quanto à possibilidade de procedimentos cirúrgicos afetarem de alguma forma o rebordo residual ${ }^{9,10,12,16}$. Cirurgias que incluem a manipulação do periósteo, em particular elevação, podem ser mais críticas com o aumento da ida$\mathrm{de}^{8,10}$.

Portanto o objetivo deste trabalho é quantificar a altura mandibular anterior após a realização de vestibuloplastia pelo método de inversão de retalhos.

\section{MATERIAL E MÉTODOS Pacientes}

Foram selecionados 13 pacientes, sendo $8 \mathrm{mu}$ lheres e 5 homens, com idades que variaram de 32 a 77 anos, com média de 54,3 anos. Esta seleção foi realizada com base nos seguintes critérios: 1) desdentados totais; 2) boas condições de saúde geral; 3) ausência de lesões de tecido mole; 4) ausência de dentes retidos, raízes residuais ou lesão óssea; 5) indicação de aprofundamento de sulco vestibular mandibular anterior; 6) altura óssea remanescente compativel com o procedimento na região anterior da mandíbula. Para o auxílio desta seleção, radiografias panorâmicas e cefalométricas foram obtidas de cada paciente*.

Verificou-se a altura óssea anterior disponivel através da radiografia cefalométrica. Para indicação da técnica de inversão de retalhos, recomenda-se altura óssea minima de $15,0 \mathrm{~mm}^{11,18}$. Nos pacientes selecionados a altura óssea média foi de $25,01 \mathrm{~mm}$, sendo a menor altura $22,0 \mathrm{~mm}$.

\section{Procedimento cirúrgico}

Foi realizado sob anestesia local, por bloqueio dos nervos alveolar inferior, lingual e bucal, bilateralmente. A técnica cirúrgica realizada foi aquela basicamente ilustrada por PICHLER; TRAUNER ${ }^{22}$ (1930) e descrita por HOWE ${ }^{11}$ (1965), com pequenas alterações, sempre realizadas por um mesmo operador.

\section{Acompanhamento radiográfico}

Os pacientes foram submetidos, como já foi mencionado anteriormente, a uma radiografia cefalométrica prévia que auxiliou na correta indicação da vestibuloplastia e que servirá para análise pré-operatória dos pacientes selecionados. Novas radiografias cefalométricas foram obtidas de cada paciente nos períodos de 30, 90 e 180 dias após a realização da cirurgia. Todas as radiografias foram realizadas por apenas um técnico e no mesmo aparelho. Os pacientes foram radiografados sem próteses e com a mandíbula em posição de repouso.

*Aparelho marca Funk, modelo Orbital X15. 
SVERZUT, C. E.; GABRIELLI, M. F. R.; VIEIRA, E. H.; SVERZUT, A. T. Avaliação radiográfica da altura mandibular anterior após vestibuloplastia por inversão de retalhos: estudo em humanos. Pesqui Odontol Bras, v. 15, n. 2, p. 133-137, abr./jun. 2001.

\section{Traçado cefalométrico}

Foram utilizadas folhas transparentes de poliéster pré-encolhido PP2910* e canetas especiais com pontas de $0,5 \mathrm{~mm}^{* *}$. As medidas foram colhidas com o auxílio de uma régua de precisão Desetec ${ }^{* * *}$.

Para nos certificarmos da correta e padronizada posição mandibular, três linhas foram traçadas. Obtiveram-se as linhas do plano mandibular (PM), násio-sela (N-S) e uma terceira utilizando-se o longo eixo da porção anterior da mandíbula (A-B). Esta linha A-B cruza com as linhas que delimitam a base da mandíbula e a porção mais superior do rebordo residual, estes pontos foram utilizados para se obter a altura mandibular anterior (AMA) (Figura 1).

\section{Análise estatística}

Os dados foram submetidos a uma análise de variância com dupla classificação: tempos e pacientes. Quando o valor de F, na análise de variância, foi significante ao nível de $5 \%$, procedeu-se ao

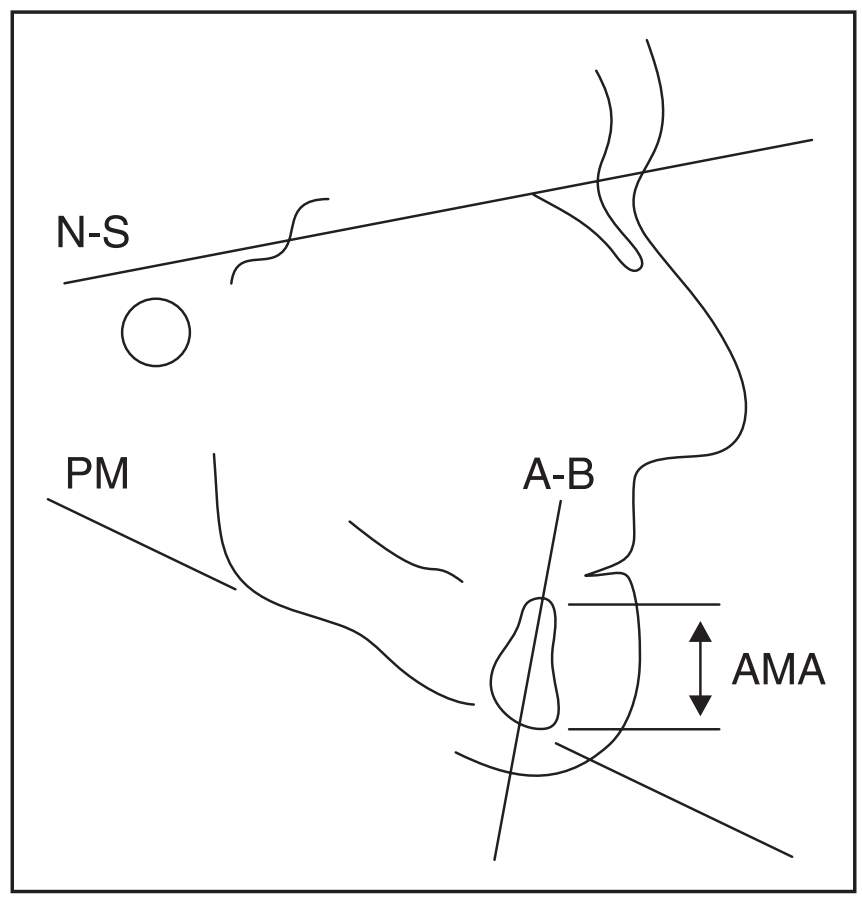

FIGURA 1 - Traçado cefalométrico: linha násio-sela (N-S), plano mandibular (PM) e linha obtida do longo eixo da porção anterior da mandíbula (A-B), da qual obteve-se o segmento da altura mandibular anterior (AMA). teste de Tukey. Estabelecemos a letra "t" para o fator "tempo", ou seja, t1 para pré-operatório, t2 para 30 dias de pós-operatório, t3 para 90 dias e t4 para 180 dias de pós-operatório.

\section{RESULTADOS}

O comportamento do fator de variação AMA (altura mandibular anterior) pode ser observado na Tabela 1.

A Tabela 1 permite observar que houve variabilidade não significativa para o fator "tempo", isto é, existiu um comportamento semelhante para as medidas do segmento AMA nos diferentes tempos, o que pode ser visualizado no Gráfico 1 .

Observa-se que a altura mandibular teve uma discreta diminuição com o tempo, porém não estatisticamente significante.

TABELA 1 - Análise de variância da AMA (altura mandibular anterior).

\begin{tabular}{l|r|c|c|c}
\hline \hline $\begin{array}{l}\text { Fonte de } \\
\text { variação }\end{array}$ & g.l. & s.q. & q.m. & $\begin{array}{c}\text { F } \\
\text { (inferior a 5\%) }\end{array}$ \\
\hline Tempo & 3 & 1,20737 & 0,402458 & 1,352 n.s. \\
\hline Paciente & 9 & 143,16693 & 15,907436 & 53,451 \\
\hline Resíduo & 17 & 5,0592940 & 0,2976055 & \\
\hline Total & 29 & 160,09467 & & \\
\hline \hline
\end{tabular}

n.s. = não significativo

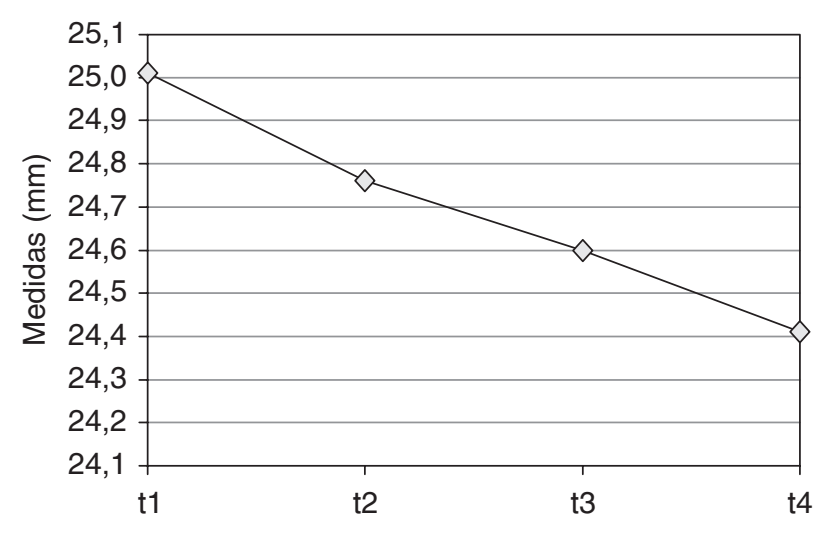

GRÁFICO 1 - Médias do segmento AMA (altura mandibular anterior). $\mathrm{t} 1=$ pré-operatório; $\mathrm{t} 2=30$ dias de pós-operatório; t3 = 90 dias de pós-operatório e t4 = 180 dias de pós-operatório.

*3M do Brasil.

** Pilot Pen do Brasil.

*** Trident do Brasil. 
SVERZUT, C. E.; GABRIELLI, M. F. R.; VIEIRA, E. H.; SVERZUT, A. T. Avaliação radiográfica da altura mandibular anterior após vestibuloplastia por inversão de retalhos: estudo em humanos. Pesqui Odontol Bras, v. 15, n. 2, p. 133-137, abr./jun. 2001.

\section{DISCUSSÃO}

A radiografia cefalométrica tem sido aceita como um método objetivo para análise de procedimentos pré-protéticos na região anterior da mandíbula ${ }^{4,6,7}$.

BOHANNAN $^{6}$ (1963), detectou uma taxa de 7\% de ampliação na imagem radiográfica nesse tipo de radiografia. Para BOLENDER; SWENSON ${ }^{7}$ (1963), esta taxa está entre 5 a $8 \%$. WATSON ${ }^{27}$ (1987), estipulou uma taxa geral de ampliação de imagem de $10 \%$, dependendo, porém, da posição de determinada estrutura em relação ao plano mediano, sendo que HILLERUP ${ }^{10}$ (1991) especificou no plano mediano uma taxa de 5,6\%.

A altura mandibular anterior (AMA), no presente estudo, sofreu uma redução média de 0,6 $\mathrm{mm}$, até os 180 dias de pós-operatório, em relação ao valor pré-operatório. Essa redução média não foi estatisticamente significativa. HILLERUP ${ }^{10}$ (1991), utilizando a técnica de inversão de retalhos, encontrou perdas que variaram de 4 a 20\%, após 2 anos de pós-operatório, medidas obtidas de telerradiografias. O valor encontrado no presente estudo corresponde a $2 \%$ da altura inicial.

Nosso trabalho revelou, no periodo analisado, perda óssea em altura de 0,6 $\mathrm{mm}$. Este valor equivale a $1,2 \mathrm{~mm} /$ ano, portanto superior à perda média em indivíduos não operados, que pode variar de $0,12 \mathrm{~mm} /$ ano a até $0,40 \mathrm{~mm} /$ ano $^{2,24,26}$. Devemos então ser criteriosos na correta indicação da técnica de vestibuloplastia por inversão de retalhos, analisando a viabilidade e o beneficio que a mesma irá trazer para o paciente e para o profissional.

Essas perdas são provavelmente relacionadas com a elevação do periósteo, procedimento que pode reduzir o aporte sangüíneo à superficie óssea ${ }^{9,10,12}$. Entretanto, mesmo com dissecção supraperiostal e enxertia de pele, a redução em altura sinfisiária tem sido descrita como de $1,05 \mathrm{~mm}$ após 1 ano de pós-operatório ${ }^{10}$. Para HILLERUP ${ }^{10}$, a reabsorção óssea é uma reação inevitável à manipulação do periósteo, sendo ele descolado do tecido ósseo ou não.

OIKARINEN ${ }^{20}$ (1965), em estudo por arteriografia da mandíbula, encontrou uma oclusão arterial parcial com o avanço da idade. Essas alterações iniciaram-se na quarta década de vida, ocorrendo a completa obliteração, em alguns casos, após a sexta década. Nos jovens, o suprimento sangüíneo mostra-se multicêntrico e centrífugo, estando basicamente sob responsabilidade da artéria alveolar inferior e secundariamente do plexo formado pelas artérias bucal, facial e lingual ${ }^{8}$. Estudos angiográficos em humanos de várias idades demonstraram obstrução da artéria alveolar inferior em $79 \%$ de todos os indivíduos estudados. Em 33\% dos pacientes, o fluxo arterial foi ausente, tendo-se associação significativa com a ausência de dentes ${ }^{8}$. Isso gera uma inversão na nutrição arterial da mandíbula, passando a ser centripeta, ou seja, o montante do abastecimento sob responsabilidade da artéria alveolar inferior declina e é compensado pelo aumento do abastecimento do plexo formado pelas artérias facial, bucal e lingual ${ }^{8}$.

Tem sido sugerido que a expansão da área de apoio de dentadura, por meio de uma vestibuloplastia, poderia reduzir a carga da prótese por unidade quadrada de osso de apoio e assim reduzir a reabsorção óssea causada pela transferência das forças oclusais ${ }^{10,16}$. Nenhuma evidência, entretanto, foi demonstrada para apoiar esse ponto de vista ${ }^{10}$.

WATSON $^{27}$ (1987) comenta que o descolamento do músculo mentoniano pode privar o tecido ósseo de estímulo funcional e que a vestibuloplastia torna chapeável uma superfície óssea não exposta previamente a forças oclusais, podendo esses dois fatores intensificar a perda óssea alveolar no período pós-cirúrgico. Portanto sugere-se que a reabsorção aumentada após vestibuloplastias pode ser decorrente de deficiência nutricional do córtex e da perda de estímulo funcional, após a elevação do periósteo.

Parece claro, portanto, que a perda do osso alveolar pode ocorrer devido a fatores sistêmicos não patológicos ${ }^{3,4,5}$. Estes não são os únicos determinantes ${ }^{1,13}$. Como salienta LASKIN ${ }^{17}$ (1987), “...a preparação de próteses satisfatórias começa com as extrações dos dentes...”. A perda traumática do osso alveolar, por causas acidentais ou iatrogênicas, pode igualar anos de reabsorção ${ }^{3}$. Por essa razão, conservação máxima do osso alveolar deve ser praticada durante a extração e tratamento dos pacientes $^{3,21}$, para que não resultem em rebordos mutilados, pois os tecidos orais são dificeis de se restituírem, uma vez perdidos ${ }^{21}$. A planificação cirúrgica prévia à construção da prótese total é um passo essencial, porém lamentavelmente, muitas vezes, isto não acontece ${ }^{17}$.

\section{CONCLUSÕES}

Ocorreu redução média na altura mandibular anterior, estatisticamente não significante, de 0,6 $\mathrm{mm}$ no período pós-operatório analisado, que totalizou 180 dias. 
SVERZUT, C. E.; GABRIELLI, M. F. R.; VIEIRA, E. H.; SVERZUT, A. T. Avaliação radiográfica da altura mandibular anterior após vestibuloplastia por inversão de retalhos: estudo em humanos. Pesqui Odontol Bras, v. 15, n. 2, p. 133-137, abr./jun. 2001.

SVERZUT, C. E.; GABRIELLI, M. F. R.; VIEIRA, E. H.; SVERZUT, A. T. Radiographic evaluation of the anterior height of the mandible after vestibuloplasty through the lipswitch technique: study in humans. Pesqui Odontol Bras, v. 15, n. 2, p. 133-137, abr./jun. 2001.

The aim of this study was to determine, through cephalometric analysis, the anterior height of the mandible after vestibuloplasty through the lipswitch technique. Thirteen patients ( 8 women and 5 men) with ages between 32 and 77 years (mean age of 54.3 years) were evaluated. The postoperative periods were 30, 90 and 180 days. During this time there was a reduction of $0.6 \mathrm{~mm}$ in the anterior height of the mandible, which was not significant.

UNITERMS: Bone resorption; Alveolar bone loss; Vestibuloplasty.

\section{REFERÊNCIAS BIBLIOGRÁFICAS}

1. ARNHEM CONSENSUS ON PREPROSTHETIC SURGERY. Int J Oral Maxillofac Surg, v. 19, p. 10-11, 1989.

2. ATWOOD, D. A. Bone loss in edentulous alveolar ridges. $\mathbf{J}$ Periodont, v. 50, p. 11-21, 1979

3. BAYS, R. A. The pathophysiology and anatomy of edentulous bone loss. In: FONSECA, R. J.; DAVIS, W. H. Reconstructive preprosthetic oral and maxillofacial surgery. Philadelphia: W. B. Saunders, 1986. p. 1-17.

4. BERGENHOLTZ, A.; PERSSON, G.; THILANDER, H. Registration technique in vestibular sulcus extension surgery in the mandible. Odontol Tidskr, v. 72, p. 197-207, 1964.

5. BILEZIKIAN, J. P.; RAISZ, L. G.; RODAN, G. A. Principles of bone biology. San Diego : Academic Press, 1996.

6. BOHANNAN, H. M. Studies in the alteration of vestibular depth. III - vestibular incision. J Periodontol, v. 34, p. 209-215, 1963.

7. BOLENDER, C.; SWENSON, R. D. Cephalometric evaluation of a labial vestibular extension procedure. J Prosthet Dent, v. 13, p. 416-431, 1963.

8. BRADLEY, J. C. The clinical significance of age changes in the vascular supply to the mandible. Int J Oral Surg, v. 10 , p. 71-76, 1981

9. FAZILI, M.; WAAS, M. A. J.; HOUVING, M. H. et al. Long-term results of vestibuloplasty of the mandible. Int J Oral Surg, v. 10, p. 77-82, 1981.

10. HILLERUP, S. Mandibular vestibuloplasty: experimental and clinical studies. Danish Med Bulletin, v. 38, p. 97-113, 1991.

11. HOWE, G. L. Preprosthetic surgery in the lower labial sulcus. Dent Pract, v. 16, p. 119-124, 1965.

12. JOOS, V.; GERNET, W.; MUZZOLINI, F. Die resorption des unterkiefers nach vestibulumplastik und mundbodensenkung. Dtsch Zahnaerz1 Z, v. 37, p. 117-120, 1982.

13. KALK, W.; VAN WAAS, M. A. J.; ENGELS, S. E. W. A comparison of different treatment strategies in patients with atrophic mandibles: a clinical evaluation after 6.5 years. Int J Prosthod, v. 5, p. 277-283, 1992.

14. KETHLEY, J. L.; GAMBLE, J. W. The lipswitch: a modification of Kazanjian's labial vestibuloplasty. J Oral Surg, v. 36, p. 701-705, 1978.

15. KWAKMAN, J. M.; VOORSMIT, R. A. C. A.; FREIHOFER, H. P. M. Treatment of the edentulous mandible with a vestibuloplasty combined with Intramobil Zylinder im- plants: a 5-year follow-up. Brit J Oral Maxillofac Surg, v. 36, p. 296-300, 1998.

16. LANDESMANN, H. M.; DAVIS, W. H.; MARTINOFF, J. et al. Resorption of the edentulous mandible after a vestibuloplasty with skin grafting. J Prosthet Dent, v. 49, p. 619-622, 1983.

17. LASKIN, D. M. Cirugia bucal y maxilo-facial. Buenos Aires : Editorial Médica Panamericana, 1987.

18. MOON, A. C. Transpositional labial vestibuloplasty of the mandible. J Can Dent Assoc, v. 18, p. 661-665, 1982.

19. NIEVES, J. W.; GOLDEN, A. L.; SIRIS, E. et al. Teenage and current calcium intake are related to bone mineral density of the hip and forearm in women aged 30-39 years. Am J Epidemiol, v. 141, p. 342-351, 1995.

20. OIKARINEN, V. J. The inferior alveolar artery - a study based on gross anatomy and arteriography, supplemented by observations on age changes. Suom Hamaslääk Toim, v. 61, p. 1-131, 1965 apud Br Dent J, v. 132, p. $142-144,1972$.

21. PETERSON, L. J.; ELLIS III, E.; HUPP, J. R. Contemporary oral and maxillofacial surgery. St. Louis : Mosby, 1988.

22. PICHLER, H.; TRAUNER, R. Die alveolarkammplastik. Z Stomatol, v. 38, p. 675-697, 1930.

23. SLEMENDA, C. W.; CHRISTIAN, J. D.; WILLIAMS, C. J. et al. Genetic determinants of bone mass in adult women: a reevaluation of the twin model and the potential importance of gene interaction on heritability estimates. $\mathbf{J}$ Bone Miner Res, v. 6, p. 561-567, 1991.

24. TALLGREN, A. The continuing reduction of the residual alveolar ridges in complete denture wearers: a mixed-longitudinal study covering 25 years. J Prosthet Dent, v. 27, p. $120-132,1972$.

25. THIES, R. W.; SAGER, R. D. Lipswitch vestibuloplasty in conjunction with implant placement. Compend Contin Educ Dent, v. 12, p. 456-463, 1991.

26. TUNCAY, O. C.; THOMSON, S.; ELLINGER, C. Cephalometric evaluation of the changes in patients wearing complete dentures. A ten-year longitudinal study. J Prosthet Dent, v. 51, p. 169-180, 1984.

27. WATSON, C. J. A radiographic analysis of a mandibular anterior vestibuloplasty with free skin graft. J Prosthet Dent, v. 58, p. 374-379, 1987.

28. WOWERN, N. V.; HJÖRTING-HANSEN, E. The mandibular bone mineral content in relation to vestibulolingual sulcoplasty. A 2-year follow-up. J Prosthet Dent, v. 65, p. 804-808, 1991.

Recebido para publicação em 27/07/00 Enviado para reformulação em 14/02/01 Aceito para publicação em 15/03/01 